\title{
Reliability analysis of two dissimilar parallel unit repairable system with failure during preventive maintenance
}

\author{
Mohit Kumar Kakkar ${ }^{\mathrm{a}^{*}}$, Ashok K Chitkara ${ }^{\mathrm{b}}$ and Jasdev Bhatti ${ }^{\mathrm{a}}$
}

${ }^{a}$ School of Mechanical Engineering, Chitkara University, Punjab, India

${ }^{b}$ Chancellor, School of Mechanical Engineering, Chitkara University, Punjab, India

\section{H R O N I C L E \\ A B S T R A C T}

Article history:

Received October 28, 2015

Received in revised format

November 28, 2015

Accepted January 28, 2016

Available online

February 1, 2016

Keywords:

Mean Time to System Failure

(MTSF)

Availability

Busy Period analysis

Profit function

\begin{abstract}
The main purpose of this research work is to present a reliability analysis of a two dissimilar parallel unit framework under the presumption that a framework's unit may also fail during the preventive maintenance (PM). Only one repair man is available with the system. It has been assumed that he is well capable of doing every repair work including preventive maintenance. Here in this work we have assumed that failure and repair times of each unit are correlated. Utilizing regenerative point strategy different reliability attributes are obtained which are very much helpful to system/ framework designers and company managers. Graphical practices of mean time to system failure and profit/cost function have been studied.
\end{abstract}

(c) 2016 Growing Science Ltd. All rights reserved.

\section{Introduction}

Reliability is defined as the ability of a device to continuously perform its intended or required function of an organization's demand without degradation or failure. These days reliability of a product is playing a very key role in the performance of the organization that is why it is receiving attention globally. It involves technique for increasing system effectiveness through reducing the frequency of failures in the system as well as maintenance cost minimization. A two-unit parallel system with one repairman has been one of the traditional models in the literature of reliability theory. Three models framework was exhibited by Kumar (1976) for a 2-unit standby system, in which Models I and II deals with a 2-unit warm-standby system under the assumption of preventive maintenance (PM) to standby unit. Here in this research work, Model III consisted a 2-unit cold-standby system with PM. In this work he gave a structure which has been superimposed on the semi-Markov processes.

* Corresponding author.

E-mail address: mohitkakkar2001@gmail.com (M. K. Kakkar) 
Dhillon and Anude (1993) presented a failure investigation of a two non-identical unit parallel system with randomly distributed repair times. In this work they, developed the equations for state probability by utilizing the variable technique. Sridharan and Mohanavadivu (1997) presented a paper on three models with general failure and they also discussed the effect of human error on reliability of a two parallel dissimilar unit system. The contrast between models I and II was that, in model I the fizzled framework was repaired back to its ordinary working state while in model II it was not. In addition, the fundamental contrast between models II and III was that, in model II it was possible for the partially failed system to be restored to its normal active state while in model III it was not. Barbera et al. (1999) presented a condition based maintenance framework with the exponential failures and settled review interims for a two-unit framework series. The state of every unit, for example, vibration or warmth, was observed at equidistant time interims. The condition pointer variables for every unit were utilized to choose whether to repair an individual unit or to update the entire framework. After the maintenance activity performed, the observed condition indicator variable takes on its initial figures. They assumed that every unit can fall flat just once inside of an investigation interim and when one or both units fizzle, the framework comes up short.

Jansen and Schouten (1995) examined the ideal preventive-upkeep plan for a generation framework comprising of $n$ - indistinguishable parallel production units. In this paper they supposed that the unit's lifetimes were IFR-distributed, also the assumed that the important expenses were because of generation misfortunes, which were expanding and arched in the quantity of units that were out of operation at the same time. Genuine maintenance expenses were thought to be immaterial when contrasted with the expenses because of those production losses.

Das and Sarkar (1999) provided an optimal preventive maintenance strategy for an industry based system. They considered an inventory system that generated a product and according to some strategy inventory was maintained. A varied demand for the product was assumed by them. They conjectured that, the down time of the system might be decreased with the help of specified preventive maintenance due to which system performance got increased. They also developed a mathematical model of the industrial system.

Levitin and Lisnianski (2000) explained the concept of optimization of preventive maintenance (PM) for multi-stage systems. This paper summed up a preventive maintenance optimization issue to multistate frameworks, which had a scope of execution levels. The dependability of framework components was characterized by their hazard functions. They concluded that appropriate activities of preventive maintenance were described by their capacity to influence the effective age of equipment. An algorithm was produced which obtained the sequence of maintenance activities providing framework functioning with the required level of reliability during its whole life by optimum maintenance expenses.

Bris et al. (2003) presented a concept which is useful to minimize the expenses of preventive maintenance (PM) applicable to series-parallel systems. In this paper, they discussed a common to all preventive maintenance (PM) model which increased the reliability to 'perfect level' which was also beneficial to minimize the maintenance expenses. Here in this work the cost function of a maintenance strategy was optimized with in some availability conditions. They also had described an algorithm for first inspection time intervals. Chandrasekhar et al. (2004) presented a cold standby system of two units. Here they assumed that the failure rate of a specific unit was steady and the repair time of that unit distribution was of two-phase Erlang distribution.

Li and Pham (2005) gave an inspection-maintenance model for industry based system including number of competing processes. In this work they developed a condition-based maintenance system with respect to the number of failure processes having degradation and impacts or shocks on that system. They had derived a long-run maintenance cost formula. A geometric sequence was used to generate the inter-inspection sequence. They also presented an algorithm to formulate the strategy that 
minimizes the maintenance cost rate which was totally depends on downhill simplex method. By using the optimization algorithm they presented some mathematical examples which explain the results.

Yao et al. (2005) discussed some policies on preventive maintenance (PM) and production. They studied the joint preventive maintenance (JPM) and production strategies for a system which was not reliable and here they also assumed that maintenance or repair time was non-negligible and stochastic. They concluded that the structure of optimal joint policies is not simple it is complex in nature. In this work they were able to characterize several properties regarding preventive maintenance including optimal production/maintenance activities etc.

A genetic algorithmic approach was presented by Sortrakul et al. (2005), in this work, heuristics based on genetic algorithms were discussed which is still useful to solve an integrated optimization framework for planning and preventive maintenance scheduling. They indicated and concluded that this presented genetic algorithm is very effective for optimizing the problem.

Dhillon and Liu (2006) Presented the effect of mistakes committed by the human beings during maintenance as found in the writing with the goal that practitioners or specialists should be very much aware of their effect and created activities to moderate their impact. This paper categorically published the work of literature and afterward examined and surveyed it deliberately. In the study presented by Wang (2007), three candidates testing procedures for series -parallel arrangement frameworks were produced taking into account the consequences of easier frameworks. The exhibitions of three candidate methodologies likewise looked at under both perspectives, one was Danger (considering only system inaccessibility) and other one was choice (considering both system unavailability and maintenance expenses) perspectives.

Zhou et al. (2007) explained reliability focused predictive maintenance arrangements for continuously observed models subject to degradation. In this work, they tried to integrate imperfect maintenance policy into condition-based predictive maintenance. They proposed this policy for a continuously observed system subject to degradation because of imperfect maintenance. They also assumed that if the system reliability reaches to a specific value an imperfect preventive maintenance (PM) was activated. They reported that imperfect preventive maintenance (PM) is important for getting optimal results.

Haggag (2009) discussed an analysis on cost factor of a system in which general failures and preventive maintenance (PM) were included. They presented a work on cost analysis of a framework by assuming that system may fail during the PM and due to some other reason. They also described that mean time to system failure was decreased with increment in the rate of failure performance of the framework also increased with the regular PM.

Kishan and Kumar (2009) showed the stochastic investigation of a two unit parallel framework model with the presumption of relationship between preventive upkeep and preventive support time. The joint appropriation of time to preventive support and preventive upkeep time was taken bivariate exponential distribution. Laggoune et al. (2010) analyzed the effect of the policy of Preventive maintenance (PM) scheduling for a multi-segment system with immediate substitution time. Hajeeh (2011) utilized the Markovian model to determine a closed form expression for the relentless state accessibility for a framework permitted to experience a few phases of deteriorations subject to several failures at each state. The failures may be of many types like random failure, common-cause failure and failure due to human error. The system was reviewed after each deteriorated state, where one of the two activities was conceivable, no activity or minimal repair which brings the system to the previous deteriorated state. He accepted that complete replacement will be permitted after $\mathrm{n}$ deteriorated states and also, the system was allowed to become as good as new after common-cause failures or failures due to human error. Nourelfath et al. (2012) detailed a joint redundancy and imperfect preventive maintenance 
planning optimization model for series-parallel multi-state degraded frameworks. Due to some financial constraints they mutually decided the maximal-accessibility series-parallel framework structure and the suitable preventive maintenance activity. System availability is defined as the ability to satisfy consumer demand that is represented as a piecewise cumulative load curve. A method was utilized, which depended on Markov procedures and universal moment generating function (UMGF), to assess the multi-state framework accessibility and the expense capacity. In this paper a heuristic approach was also presented by the authors to solve the formulated and mathematical problem.

Xia et al. (2012) created multi-attribute model (MAM) which was used to find the minimum maintenance time intervals. At that point, a combination of series-parallel of the framework was examined as far as the whole-system schedule is concerned. Maintenance time window (MTW) programming was exhibited to make a cost-effective/practical framework schedule by progressively using maintenance opportunities. The maintenance scheme obtained by using the proposed MAMMTW strategy was exhibited through a contextual investigation in a hydraulic steering manufacturing plant. Here in this work they had concluded that regular focus on maintenance tends to a significant cost reduction. In the research paper of Hajeeh (2012) the execution of a framework that was upon failure could experience one of a few repairs; imperfect repair, minimal repair, or replacement (perfect repair), was analyzed and examined. An analytical expression for the accessibility and reliability of the general system and three special cases were derived along with the long run probabilities of the distinctive conditions of the framework. A case was additionally exhibited to outline or illustrate and compare the performance of the three cases. Distefano et al. (2013) discussed the latest treatment of advanced analytic, state-space based techniques to study reliability systems with distributions other than exponential. They gave an outline of distinctive strategies for the solution of non-Markovian statespace based models, including phase-type expansion and structure which permits us to manage reestablishment with renewal, semi-Markov, and Markov-regenerative processes, trying to characterize them on Reliability and dependability connections. In the last section of this work, they explained these techniques with the assistance of a few examples dealing with general non-exponential reliability behaviors. Wong (2013) studied the preventive support models for stochastically breaking down singleunit frameworks. Doostparast et al. (2014) discussed the issue of reliability-based periodic preventive maintenance (PPM) planning for frameworks with deteriorating components. The fundamental motivation behind this was to keep up a certain level of reliability with minimal total maintenance related cost. It was expected that the arbitrary disappointments take after a Non-Homogeneous Poisson Process. Ruiz-Castro (2015) talked about the PM Policies and arrangements for a standby framework and its impact on the framework's availability of a general k-out-of-n: G System with dissimilar components under the assumption of shut-Off procedures having Quasi-Birth-Death (QBD) Process.

All the above studies assumed that during preventive maintenance no unit is going to fail but in real situation this fact does not hold good. Preventive Maintenance plays a key role in the field of reliability engineering. It is likewise a well-known certainty that an essentially huge extent of failures occur during the maintenance phase. Failures during maintenance are a subject which in the past has not been given the amount of consideration that it merits. So our work will be very beneficial to system designers and engineers working in the area of Preventive Maintenance (PM). Keeping the above circumstances in perspective we examine a two unit non identical parallel system introducing the concept of failure of unit during Preventive maintenance. Measures of system execution or performance such as reliability, mean time to system failure (MTSF), system availability, and steady-state availability are also determined which are exceptionally helpful to framework engineers, architects and designers.

\section{System model description and assumptions}

This system consists of two dissimilar units (unit-A and unit-B). Initially, system starts its operations from state $\mathrm{S}_{0}$ in which unit- $\mathrm{A}$ and $\mathrm{B}$ both are operative. 
b) In state $S_{0}$, unit $A$ and $B$ both are in active mode.

c) In state $\mathrm{S}_{1}$, unit $\mathrm{A}$ is in active mode but $\mathrm{B}$ is in failure mode.

d) In state $\mathrm{S}_{2}$, unit $\mathrm{B}$ is in active mode but $\mathrm{A}$ is in failure mode.

e) In state $S_{3}$, unit $A$ is in active mode but $B$ is under PM.

f) In state $\mathrm{S}_{4}$, unit $\mathrm{B}$ is in active mode but $\mathrm{A}$ is under PM.

g) In state $\mathrm{S}_{5}$, unit $\mathrm{A}$ and $\mathrm{B}$ both are in failure mode( but $\mathrm{A}$ is waiting for repair man).

h) In state $\mathrm{S}_{6}$, unit $\mathrm{A}$ and $\mathrm{B}$ both are in failure mode( but B is waiting for repair man).

\section{Notations and Transition Diagram:}

$\theta_{1}: \quad$ Constant rate of unit A going for PM

$\theta_{2}$ : Constant rate of unit B going for PM

$\omega_{1}: \quad$ Constant rate of PM for unit A

$\omega_{2}: \quad$ Constant rate of Pm for unit B

$X_{i}$ :

$f_{i}(x, y)$ :

Random variables representing the failure times of $\mathrm{A}$

Joint pdf of $\left(\mathrm{x}_{\mathrm{i}}, \mathrm{y}_{\mathrm{i}}\right) ; \mathrm{i}=1,2$

$=\alpha_{i} \beta_{i}\left(1-r_{i}\right) e^{-\alpha_{i} x-\beta_{i} y} I_{0}\left(2 \sqrt{\left(\alpha_{i} \beta_{i} r_{i} x y\right.}\right) ; X, Y, \alpha_{i}, \beta_{i}>0 ; 0 \leq r_{i}<1$,

where $I_{0}\left(2 \sqrt{\alpha_{i} \beta_{i} r_{i} x y}\right)=\sum_{j=0}^{\infty} \frac{\left(\alpha_{i} \beta_{i} r_{i} x y\right)^{j}}{(j !)^{2}}$

$\mathrm{k}_{\mathrm{i}}(\mathrm{Y} / \mathrm{X})$ : $\quad$ Conditional pdf of $\mathrm{Y}_{\mathrm{i}}$ given $\mathrm{X}_{\mathrm{i}}=\mathrm{x}$ is given by

$=\beta_{i} e^{-\alpha_{i} r_{i} x-\beta_{i} y} I_{0}\left(2 \sqrt{\left(\alpha_{i} \beta_{i} r_{i} x y\right.}\right)$

$\mathrm{g}_{\mathrm{i}}($.$) : \quad$ Marginal pdf of $\mathrm{X}_{\mathrm{i}}=\alpha_{i}\left(1-r_{i}\right) e^{-\alpha_{i}\left(1-r_{i}\right) x}$

$\mathrm{h}_{\mathrm{i}}($.$) : \quad$ Marginal pdf of $\mathrm{Y}_{\mathrm{i}}=\beta_{i}\left(1-r_{i}\right) e^{-\beta_{i}\left(1-r_{i}\right) y}$

$q_{i j}(),. Q_{i j}():. \quad \quad$ pdf $\&$ cdf of transition time from regenerative states pdf $\&$ cdf of transition time from regenerative state $S_{i}$ to $S_{j}$.

$\mathrm{x} \quad$ Probability of failure of unit A during PM

y Probability of not failure of unit A during PM

$\mathrm{p} \quad$ Probability of failure of unit B during PM

q Probability of not failure of unit B during PM

$\mu_{i}: \quad$ Mean sojourn time in state $\mathrm{S}_{\mathrm{i}}$.

$\oplus: \quad$ Symbol of ordinary Convolution $\quad A(t) \oplus B(t)=\int_{0}^{t} A(t-u) B(u) d u$

$\otimes:$

Symbol of stieltjes convolution $A(t) \otimes B(t)=\int_{0}^{t} A(t-u) d B(u)$ 


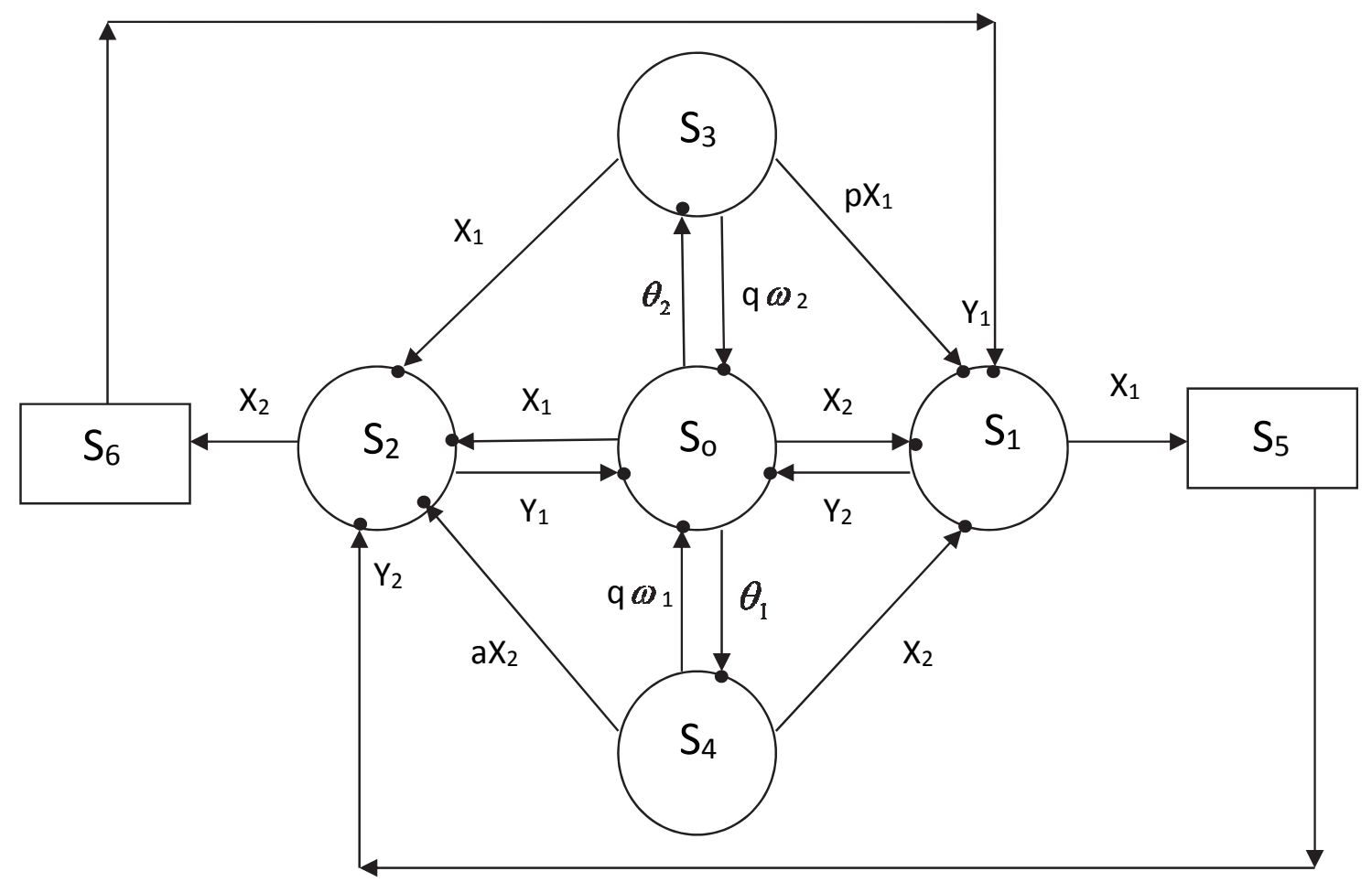

Fig. 1. Transition Diagram

\subsection{Transition probability and sojourn times:}

The steady state transition probability can be as follows:

$$
\begin{aligned}
& p_{01}=\frac{\alpha_{2}\left(1-r_{2}\right)}{\theta_{1}+\theta_{2}+\alpha_{2}\left(1-r_{2}\right)+\alpha_{1}\left(1-r_{1}\right)} \\
& p_{02}=\frac{\alpha_{1}\left(1-r_{1}\right)}{\theta_{1}+\theta_{2}+\alpha_{2}\left(1-r_{2}\right)+\alpha_{1}\left(1-r_{1}\right)} \\
& p_{03}=\frac{\theta_{2}}{\theta_{1}+\theta_{2}+\alpha_{2}\left(1-r_{2}\right)+\alpha_{1}\left(1-r_{1}\right)} \\
& p_{04}=\frac{\theta_{1}}{\theta_{1}+\theta_{2}+\alpha_{2}\left(1-r_{2}\right)+\alpha_{1}\left(1-r_{1}\right)} \\
& p_{10}=\frac{\beta_{2}\left(1-r_{2}\right)}{\beta_{2}\left(1-r_{2}\right)+\alpha_{1}\left(1-r_{1}\right)} \\
& p_{15}=p_{12.5}=\frac{\alpha_{1}\left(1-r_{1}\right)}{\beta_{2}\left(1-r_{2}\right)+\alpha_{1}\left(1-r_{1}\right)} \\
& p_{20}=\frac{\beta_{1}\left(1-r_{1}\right)}{\beta_{1}\left(1-r_{1}\right)+\alpha_{2}\left(1-r_{2}\right)} \\
& p_{26}=p_{21.6}=\frac{\alpha_{2}\left(1-r_{2}\right)}{\beta_{1}\left(1-r_{1}\right)+\alpha_{2}\left(1-r_{2}\right)} \\
& p_{30}=\frac{q \omega_{2}}{q \omega_{2}+\alpha_{1}\left(1-r_{1}\right)+p \alpha_{2}\left(1-r_{2}\right)}
\end{aligned}
$$




$$
\begin{aligned}
& p_{31}=\frac{p \alpha_{2}\left(1-r_{2}\right)}{q \omega_{2}+\alpha_{1}\left(1-r_{1}\right)+p \alpha_{2}\left(1-r_{2}\right)} \\
& p_{32}=\frac{\alpha_{1}\left(1-r_{1}\right)}{q \omega_{2}+\alpha_{1}\left(1-r_{1}\right)+p \alpha_{2}\left(1-r_{2}\right)} \\
& p_{40}=\frac{y \omega_{1}}{y \omega_{1}+x \alpha_{1}\left(1-r_{1}\right)+\alpha_{2}\left(1-r_{2}\right)} \\
& p_{41}=\frac{\alpha_{2}\left(1-r_{2}\right)}{y \omega_{1}+x \alpha_{1}\left(1-r_{1}\right)+\alpha_{2}\left(1-r_{2}\right)} \\
& p_{42}=\frac{x \alpha_{1}\left(1-r_{1}\right)}{y \omega_{1}+x \alpha_{1}\left(1-r_{1}\right)+\alpha_{2}\left(1-r_{2}\right)}
\end{aligned}
$$

Here we can see that

$$
\begin{aligned}
& p_{01}+p_{02}+p_{03}+p_{04}=1 \\
& p_{10}+p_{15}=1 \\
& p_{20}+p_{26}=1 \\
& p_{30}+p_{31}+p_{32}=1 \\
& p_{40}+p_{41}+p_{42}=1
\end{aligned}
$$

\section{Mean sojourn time}

The unconditional mean time taken by the system to transit for any state $\mathrm{j}$ when it is counted from epoch of entrance into state i mathematically stated as:

$$
m_{i j}=\int_{0}^{\infty} t q_{i j}(t) d t=-q_{i j}^{* \prime}(0)
$$

\section{Analysis of Characteristics}

\subsection{MTSF (Mean Time to System Failure)}

To determine the MTSF of the system, we regard the failed state of the system as absorbing state, by probabilistic arguments, we get

$$
\begin{aligned}
& \phi_{0}(t)=Q_{01}(t) \otimes \phi_{1}(t)+Q_{02}(t) \otimes \phi_{2}(t)+Q_{03}(t) \otimes \phi_{3}(t)+Q_{04}(t) \otimes \phi_{4}(t) \\
& \phi_{1}(t)=Q_{10}(t) \otimes \phi_{0}(t)+Q_{15}(t) \\
& \phi_{2}(t)=Q_{20}(t) \otimes \phi_{0}(t)+Q_{26}(t) \\
& \phi_{3}(t)=Q_{30}(t) \otimes \phi_{0}(t)+Q_{31}(t) \otimes \phi_{1}(t)+Q_{32}(t) \otimes \phi_{2}(t) \\
& \phi_{4}(t)=Q_{40}(t) \otimes \phi_{0}(t)+Q_{41}(t) \otimes \phi_{1}(t)+Q_{42}(t) \otimes \phi_{2}(t)
\end{aligned}
$$


Taking Laplace Stieltjes transforms of these relations and solving for $\phi_{0}^{* *}(s)$,

$$
\begin{aligned}
& \phi_{0}^{* *}(s)=\frac{N(s)}{D(s)}, \\
& \mathrm{MTSF}=\lim _{s \rightarrow o} \frac{\left(1-\phi_{0}^{* *}(s)\right)}{s}=\frac{N}{D}
\end{aligned}
$$

After taking the limit with the help of L-Hospital's rule we have

$$
\begin{aligned}
& \left.N=\mu_{0}+\mu_{1}\left(p_{01}+p_{31} p_{03}+p_{04} p_{41}\right)+\mu_{2}\left(\mathrm{p}_{02}+\mathrm{p}_{03} \mathrm{p}_{32}+p_{04} p_{42}\right)\right)+\mu_{3} \mathrm{p}_{03}+\mu_{4} \mathrm{p}_{04} \\
& D=p_{01} p_{15}+\mathrm{p}_{15} p_{03} p_{31}+\mathrm{p}_{04} \mathrm{p}_{15} p_{41}+\mathrm{p}_{02} \mathrm{p}_{26}+p_{03} \mathrm{p}_{26} \mathrm{p}_{32}+p_{04} p_{42} p_{26}
\end{aligned}
$$

\subsection{Availability Analysis}

Let $A_{i}(t)$ be the probability that the system is in up-state at instant $t$ given that the system entered regenerative state $i$ at $t=0$. Using the arguments of the theory of a regenerative process the point wise availability $A_{i}(t)$ is seen to satisfy the following recursive relations

$$
\begin{aligned}
& A_{0}(t)=M_{0}(t)+q_{01}(t) \oplus A_{1}(t)+q_{02}(t) \oplus A_{2}(t)+q_{03}(t) \oplus A_{3}(t)+q_{04}(t) \oplus A_{4}(t) \\
& A_{1}(t)=M_{1}(t)+q_{10}(t) \oplus A_{0}(t)+q_{12.5}(t) \oplus A_{2}(t) \\
& A_{2}(t)=M_{2}(t)+q_{20}(t) \oplus A_{0}(t)+q_{21.6}(t) \oplus A_{1}(t) \\
& A_{3}(t)=M_{3}(t)+q_{30}(t) \oplus A_{0}(t)+q_{31}(t) \oplus A_{1}(t)+q_{32}(t) \oplus A_{2}(t) \\
& A_{4}(t)=M_{4}(t)+q_{40}(t) \oplus A_{0}(t)+q_{41}(t) \oplus A_{1}(t)+q_{42}(t) \oplus A_{2}(t)
\end{aligned}
$$

where

$$
A_{0}^{*}(s)=\frac{N_{1}(s)}{D_{1}(s)}
$$

The steady state availability is

$$
A_{0}=\lim _{s \rightarrow o} s A_{0}^{*}(s)=\frac{N_{1}}{D_{1}}
$$

where

$$
\begin{aligned}
& N_{1}=\mu_{0}\left(1-p_{12.5} p_{26}\right)+\mu_{1}\left(p_{12.5} p_{26} p_{42}+p_{41} p_{04}+p_{03} p_{26} p_{32}+p_{o 3} p_{31}+p_{01}+p_{02} p_{51}\right) \\
& +\mu_{2}\left(p_{42} p_{04}+p_{04} p_{41} p_{26}+p_{32} p_{03}+p_{12.5} p_{31}\right)-\mu_{3}\left(p_{03}+p_{26} p_{30}\right) \\
& +\mu_{4}\left(p_{41}+p_{26} p_{12.5} p_{04}\right) \\
& D_{1}=\mu_{0}\left(1-p_{10} p_{26}-p_{20}\right)+\mu_{1}\left(p_{03}+p_{03} p_{31}+p_{01}+p_{02} p_{26}\right) \\
& +\mu_{2}\left(p_{04} p_{42} p_{10}+p_{10} p_{03}+p_{03} p_{15}\right)+\mu_{3}\left(p_{10}+p_{20}-p_{10} p_{20}\right)+\mu_{4}
\end{aligned}
$$

\subsection{Busy Period Analysis of the Repairman}

Let $\mathrm{B}_{\mathrm{i}}(\mathrm{t})$ be the probability that the repairman is busy at instant $t$, given that the system is entered to regenerative state $i$ at $\mathrm{t}=0$. By probabilistic arguments, we have the following recursive relations for $B_{i}(t)$. 
$B_{0}(t)=q_{01}(t) \oplus B_{1}(t)+q_{02}(t) \oplus B_{2}(t)+q_{03}(t) \oplus B_{3}(t)+q_{04}(t) \oplus B_{4}(t)$

$B_{1}(t)=W_{1}(t)+q_{10}(t) \oplus B_{0}(t)+q_{12.5}(t) \oplus B_{2}(t)$

$B_{2}(t)=W_{2}(t)+q_{20}(t) \oplus B_{0}(t)+q_{21.6}(t) \oplus B_{1}(t)$

$B_{3}(t)=W_{3}(t)+q_{30}(t) \oplus B_{0}(t)+q_{31}(t) \oplus B_{1}(t)+q_{32}(t) \oplus B_{2}(t)$

$B_{4}(t)=W_{4}(t)+q_{40}(t) \oplus B_{0}(t)+q_{41}(t) \oplus B_{1}(t)+q_{42}(t) \oplus B_{2}(t)$

Now taking Laplace transform of these equations and

solving them for $B_{0}^{*}(s)$, we get

$B_{0}^{*}(s)=\frac{N_{2}(s)}{D_{1}(s)}$

The steady state availability is

$$
\begin{aligned}
& \quad B_{0}=\lim _{s \rightarrow o} s B_{0}^{*}(s)=\frac{N_{2}}{D_{1}} \\
& N_{2}=\mu_{1}\left(p_{04} p_{26} p_{42}-\mathrm{p}_{41} \mathrm{p}_{04}+\mathrm{p}_{03} \mathrm{p}_{26} \mathrm{p}_{32}+\mathrm{p}_{03} \mathrm{p}_{31}+\mathrm{p}_{01}+p_{02} \mathrm{p}_{26}\right) \\
& +\mu_{2}\left(-\mathrm{p}_{04} \mathrm{p}_{42}-p_{15} \mathrm{p}_{04} p_{41}+\mathrm{p}_{03} \mathrm{p}_{32}\left(1+p_{15}\right)+\mathrm{p}_{01} \mathrm{p}_{15}+p_{02}\right) \\
& +\mu_{3}\left(\mathrm{p}_{03}\right)\left(1-p_{12.5} p_{26}\right)-\mu_{4} \mathrm{p}_{04}\left(1-\mathrm{p}_{12.5} \mathrm{p}_{26}\right) \\
& \mathrm{D}_{1} \text { has already been specified. }
\end{aligned}
$$

\subsection{Expected Number of Visits by the Repairman}

We define $V_{i}(t)$ as the expected number of visits by the repairman in $(0, \mathrm{t}]$, given that the system initially starts from regenerative state $S_{i}$. By probabilistic arguments we have the following recursive relations for $V_{i}(t)$

$$
\begin{aligned}
& V_{0}(t)=Q_{01}(t) \otimes\left(1+V_{1}(t)\right)+Q_{02}(t) \otimes\left(1+V_{2}(t)\right)+Q_{03}(t) \otimes\left(1+V_{3}(t)\right)+Q_{04}(t) \otimes\left(1+V_{4}(t)\right) \\
& V_{1}(t)=Q_{10}(t) \otimes V_{0}(t)+Q_{12.5}(t) \otimes V_{2}(t) \\
& V_{2}(t)=Q_{20}(t) \otimes V_{0}(t)+Q_{21.6}(t) \otimes V_{1}(t) \\
& V_{3}(t)=Q_{30}(t) \otimes V_{0}(t)+Q_{31}(t) \otimes V_{1}(t)+Q_{32}(t) \otimes V_{2}(t) \\
& V_{4}(t)=Q_{40}(t) \otimes V_{0}(t)+Q_{41}(t) \otimes V_{1}(t)+Q_{42}(t) \otimes V_{2}(t)
\end{aligned}
$$

Taking Laplace stieltjes transform of the equations of expected number of visits and solving them for $V_{0}^{* *}(s)$ yields

$$
V_{0}=\lim _{s \rightarrow o} s V_{0}^{*}(s)=\frac{N_{3}}{D_{1}}
$$

In steady state

$$
\begin{aligned}
& V_{0}=\lim _{s \rightarrow o} s V_{0}^{*}(s)=\frac{N_{3}}{D_{1}} \\
& N_{3}=\mu_{0}\left(1-p_{12.5} p_{26}\right)+p_{04}\left(\mu_{1} p_{03} p_{26} p_{32}+\mu_{2} p_{o 3} p_{31}+\mu_{3} p_{02} p_{26}\right)
\end{aligned}
$$

$\mathrm{D}_{1}$ has already been specified.

\section{Profit analysis and conclusion}

The expected total profit incurred to the system in steady state is given by 


$$
P=C_{0} A_{0}-C_{1} B_{0}-C_{2} V_{0}
$$

where

$C_{0}=$ Revenue/unit up time of the system,

$C_{1}=$ Cost/unit time for which repairman is busy,

$C_{2}=$ Cost $/$ visit of repairman,

For a more clear view of the system characteristics w.r.t. the various parameters involved, we plot curves for MTSF and Availability function in Fig.2 and Fig.3 w.r.t the failure parameter $(\alpha)$ of unit A for three different values of correlation coefficient, between $\mathrm{X}$ and $\mathrm{Y}$, while the other parameters are kept fixed as

$$
\begin{aligned}
& \theta_{1}=0.001, \theta_{2}=0.0005, \omega_{1}=0.001, \omega_{2}=0.0002, p=0.2, q=1-p, x=0.2, y=1-x, r_{2}=0.3 \\
& \alpha_{2}=0.5, \beta_{1}=0.01, \beta_{2}=0.02, C_{0}=1500, C_{1}=500, C_{2}=50
\end{aligned}
$$

From the Fig. 2 it is clear that availability decreases as failure rate increases. Also for the fixed value of failure rate, the availability is higher for high correlation (r). From the Fig. 3 it is observed that MTSF decreases as failure rate increases irrespective of other parameters. The curves also indicates that for the same value of failure rate, MTSF is higher for higher values of correlation coefficient(r), so here we conclude that the high value of $r$ tends to increase the expected life time of the system. From the Fig. 3 it is clear that availability decreases as failure rate increases. Also for the fixed value of failure rate, the availability is higher for high correlation (r). Fig. 4 shows the trend of busy period of the repairman of the system with respect to failure rate of unit for different values of correlation coefficient(r). It is clear from the trend of the graph in the figure that busy period of single repairman increases with increment in the values of the failure rate and it is higher for the higher values of the correlation coefficient(r). Fig. 5 describes that as the failure rate is moving right direction as we can easily see the downfall of profit curve but again we are getting the same trend of the three curves with respect to the correlation coefficient as for a fixed failure rate profit is higher for greater correlation coefficient " $r$ ". It can be concluded from the graph that the profit gets increased with an increase in the values of correlation coefficients, but get decreased with the higher values of failure rate. For an example we can see in the following Table 1 that for a fixed value of correlation coefficient MTSF, availability and profit all are decreasing with the increase of failure rate.

\begin{tabular}{|c|c|c|c|c|}
\hline Failure rate & Correlation Coefficient & MTSF & Availability & Profit \\
\hline 0.11 & \multirow{5}{*}{$\mathrm{r}_{11}=0.25$} & 1.029708 & 0.452254 & 8608.941 \\
\hline 0.12 & & 1.029723 & 0.436274 & 8337.789 \\
\hline 0.13 & & 1.029778 & 0.422633 & 8164.097 \\
\hline 0.14 & & 1.029857 & 0.410852 & 7983.121 \\
\hline 0.15 & & 1.029953 & 0.400572 & 7823.329 \\
\hline 0.11 & \multirow{5}{*}{$\mathrm{r}_{12}=0.50$} & 1.031805 & 0.464331 & 8768.118 \\
\hline 0.12 & & 1.031552 & 0.442462 & 8434.823 \\
\hline 0.13 & & 1.031391 & 0.42374 & 8142.673 \\
\hline 0.14 & & 1.031298 & 0.407531 & 7884.53 \\
\hline 0.15 & & 1.031254 & 0.393359 & 7654.811 \\
\hline 0.11 & \multirow{5}{*}{$\mathrm{r}_{13}=0.75$} & 1.038655 & 0.615493 & 11873.71 \\
\hline 0.12 & & 1.037572 & 0.579002 & 11333.18 \\
\hline 0.13 & & 1.036688 & 0.547413 & 10849.43 \\
\hline 0.14 & & 1.035963 & 0.519815 & 10414 \\
\hline 0.15 & & 1.035368 & 0.495504 & 10020.03 \\
\hline
\end{tabular}

\section{Table 1}

The results of different values of MTSF, Availability and Profit under different failure rate

This paper helps us in analyzing the reliability characteristics like MTSF, Availability profit, busy period etc. these graphs are very much beneficial to industrial people and engineers engaged for the system in industry because with the help of these graphs the cut-off points of the failure rates, the profit of the system can be analyzed. Also, various rates/revenue per unit up time/costs can be obtained which help in deciding the upper/lower acceptable values of rates/costs so that the system is cost-effective. 
Other Graphs can be plotted and then various conclusions can be observed/derived which will be very useful in taking various decisions beneficial to the system analyst or managers.

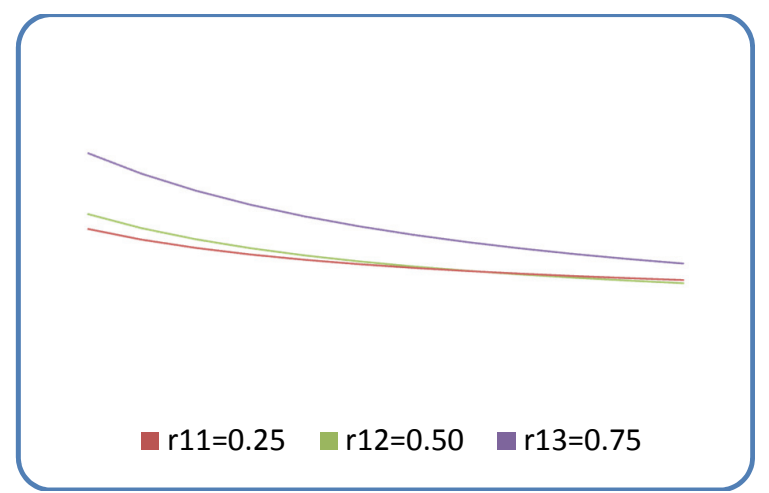

Fig. 2. Availability vs Failure Rate

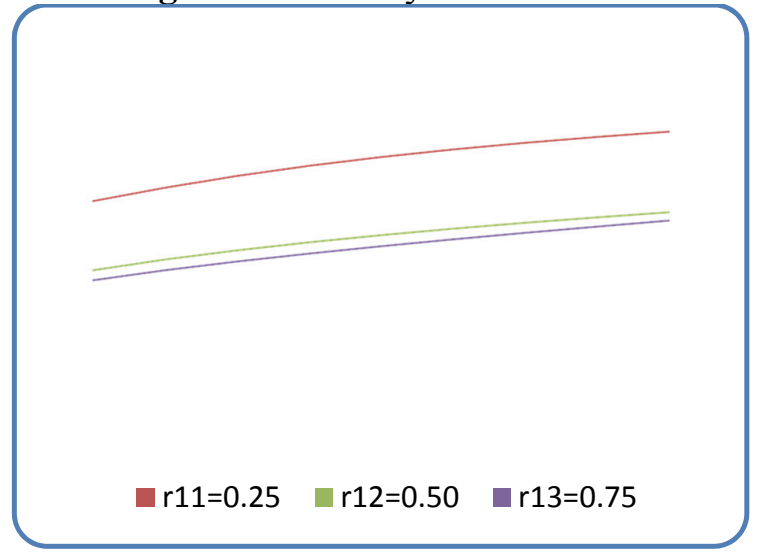

Fig. 4. Busy period vs Failure Rate

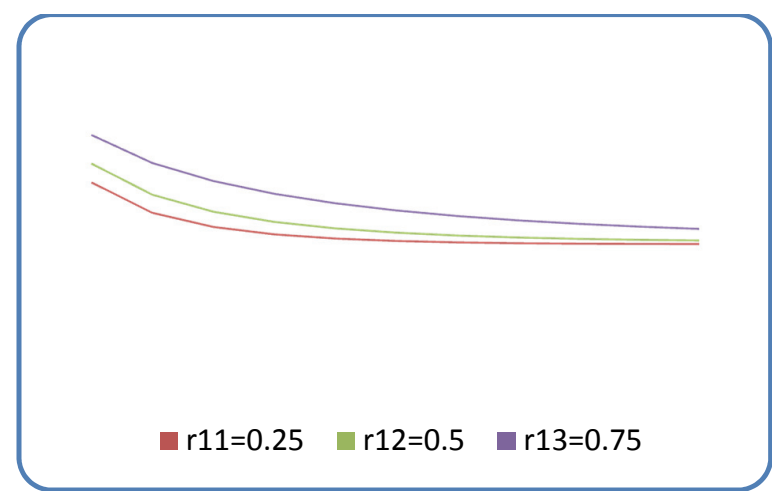

Fig. 3. MTSF vs Failure Rate

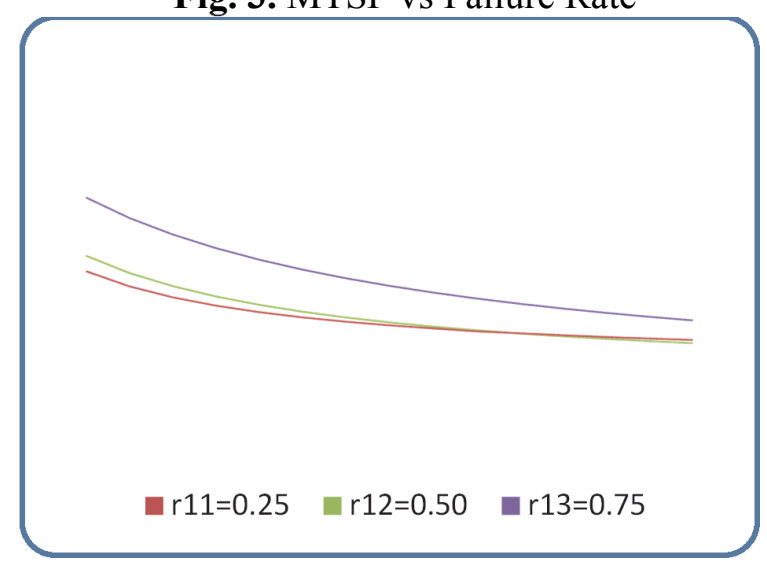

Fig. 5. Profit vs Failure Rate

\section{References}

Barbera, F., Schneider, H., \& Watson, E. (1999). A condition based maintenance model for a two-unit series system. European Journal of Operational Research, 116(2), 281-290.

Bris, R., Châtelet, E., \& Yalaoui, F. (2003). New method to minimize the preventive maintenance cost of series-parallel systems. Reliability Engineering \& System Safety, 82(3), 247-255.

Chandrasekhar, P., Natarajan, R., \& Yadavalli, V. S. S. (2004). A study on a two unit standby system with Erlangian repair time. Asia-Pacific Journal of Operational Research, 21(03), 271-277.

Das, T. K., \& Sarkar, S. (1999). Optimal preventive maintenance in a production inventory system. IIE Transactions, 31(6), 537-551.

Distefano, S., \& Trivedi, K. S. (2013). Non-Markovian State-Space Models in Dependability Evaluation. Quality and Reliability Engineering International, 29(2), 225-239.

Dhillon, B. S., \& Anude, O. C. (1993). Common-cause failure analysis of a non-identical unit parallel system with arbitrarily distributed repair times. Microelectronics Reliability, 33(1), 87-103.

Dhillon, B. S., \& Liu, Y. (2006). Human error in maintenance: a review. Journal of Quality in Maintenance Engineering, 12(1), 21-36.

Doostparast, M., Kolahan, F., \& Doostparast, M. (2014). A reliability-based approach to optimize preventive maintenance scheduling for coherent systems. Reliability Engineering \& System Safety, 126, 98-106.

Gertsbakh, I. (2000). Reliability theory: with applications to preventive maintenance. Springer Science \& Business Media. 
Haggag, M. Y. (2009). Cost analysis of a system involving common-cause failures and preventive maintenance. Journal of Mathematics and Statistics,5(4), 305.

Hajeeh, M. A. (2011). Availability of deteriorated system with inspection subject to common-cause failure and human error. International Journal of Operational Research, 12(2), 207-222.

Hajeeh, M. A. (2012). Availability of a system with different repair options. International Journal of Mathematics in Operational Research, 4(1), 41-55.

Jansen, J., Schouten, F. V. D. D. (1995). Maintenance optimization on parallel production units. IMA Journal of Management Mathematics, 6(1), 113-134.

Kishan, R., \& Kumar, M. (2009). Stochastic analysis of a two-unit parallel system with preventive maintenance. Journal of Reliability and Statistical Studies, 22, 31-38.

Kumar, A. (1976). Steady-state profit in a 2-unit standby system. Reliability, IEEE Transactions on, 25(2), 105-108.

Laggoune, R., Chateauneuf, A., \& Aissani, D. (2010). Preventive maintenance scheduling for a multicomponent system with non-negligible replacement time. International Journal of Systems Science, 41(7), 747-761.

Levitin, G., \& Lisnianski, A. (2000). Optimization of imperfect preventive maintenance for multi-state systems. Reliability Engineering \& System Safety, 67(2), 193-203.

Li, W., \& Pham, H. (2005). An inspection-maintenance model for systems with multiple competing processes. Reliability, IEEE Transactions on, 54(2), 318-327.

Ruiz-Castro, J. E. (2015). A preventive maintenance policy for a standby system subject to internal failures and external shocks with loss of units. International Journal of Systems Science, 46(9), 1600-1613.

Sridharan, V., \& Mohanavadivu, P. (1997). Reliability and availability analysis for two non-identical unit parallel systems with common cause failures and human errors. Microelectronics Reliability, $37(5), 747-752$.

Sortrakul, N., Nachtmann, H. L., \& Cassady, C. R. (2005). Genetic algorithms for integrated preventive maintenance planning and production scheduling for a single machine. Computers in Industry, 56(2), 161-168.

Nourelfath, M., Châtelet, E., \& Nahas, N. (2012). Joint redundancy and imperfect preventive maintenance optimization for series-parallel multi-state degraded systems. Reliability Engineering \& System Safety, 103, 51-60.

Wang, M. (2007). Testing strategies for parallel-series standby systems. In Industrial Engineering and Engineering Management, 2007 IEEE International Conference on (pp. 645-648). IEEE.

Wong, C. S., Chan, F. T. S., \& Chung, S. H. (2013). A joint production scheduling approach considering multiple resources and preventive maintenance tasks. International Journal of Production Research, 51(3), 883-896.

Xia, T., Xi, L., Zhou, X., \& Lee, J. (2012). Dynamic maintenance decision-making for series-parallel manufacturing system based on MAM-MTW methodology. European Journal of Operational Research, 221(1), 231-240.

Yao, X., Xie, X., Fu, M. C., \& Marcus, S. I. (2005). Optimal joint preventive maintenance and production policies. Naval Research Logistics (NRL), 52(7), 668-681.

Zacks, S. (2012). Introduction to reliability analysis: probability models and statistical methods. Springer Science \& Business Media.

Zhou, X., Xi, L., \& Lee, J. (2007). Reliability-centered predictive maintenance scheduling for a continuously monitored system subject to degradation.Reliability Engineering \& System Safety, 92(4), 530-534.

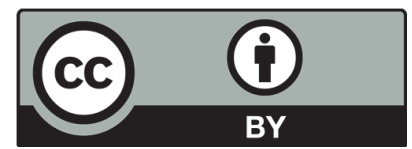

(C) 2016 by the authors; licensee Growing Science, Canada. This article is an open access article distributed under the terms and conditions of the Creative Commons Attribution (CC-BY) license (http://creativecommons.org/licenses/by/4.0/). 